\title{
Cylindrical Geometry: A Further Step in Active Microwave Tomography
}

\author{
Antoni Broquetas, Member, IEEE, Jordi Romeu, Student Member, IEEE, Juan M. Rius, Student \\ Member, IEEE, Antonio R. Elias-Fuste, Member, IEEE, Angel Cardama, Member, IEEE, \\ and Luis Jofre, Member, IEEE
}

\begin{abstract}
A prototype imaging system for active microwave tomography using cylindrical geometry has been developed, making is possible to obtain images of the dielectric properties of biological targets at $2.45 \mathrm{GHz}$. The system requires no mechanical movements to illuminate the body from multiple directions (views) and measure the scattered fields. In this way a complete data set consisting in 64 views is acquired in $3 \mathrm{~s}$ using low-power illumination. The system is described, including images obtained with biological phantoms and actual bodies.
\end{abstract}

\section{INTRODUCTION}

C UURENT research in medical imaging is devoted both to improving existing systems based on $\mathrm{X}$ rays, nuclear magnetic resonance, ultrasound, etc., and to investigating other emerging techniques such as applied potential imaging [1] and microwave imaging [2]. The interest in developing new imaging methods when very good ones already exist lies basically in the different physical parameters visualized, which depend strongly on the type of radiation used and its interaction with biological materials.

The objective of active microwave tomography is to reconstruct the dielectric properties of a body illuminated with microwaves from a measurement of the scattered fields. Biological tissues present large variations in their electrical characteristics depending on composition, temperature, irrigation, etc.; therefore their internal structure and physiological changes can in principle be imaged [3], [4]. Unlike ionizing radiation, low-power microwave radiation allows a virtually safe exploration of living tissues; thus even continuous monitoring can be envisaged. Moreover the technology involved is common to radar and communication systems; thus it is well developed and inexpensive.

Some drawbacks of this technique are its low resolution (of the order of a wavelength of the radiation in tissue),

Manuscript received July 23, 1990; revised December 17, 1990. This work was supported by the Spanish Committee for Scientific and Technical Research (CAICYT) under Grant $1165-84$, by the Spanish National Institute of Health (FISS) under Grant $84 / 2112$, by the Spanish-French Cooperation Program under Grant $30 / 135$, and by the Spanish-British Cooperation Program under Grant 17/173.

The authors are with the TSC Dept., Group AMR, Universitat Politècnica de Catalunya, P.O. Box 30002, 08080 Barcelona, Spain.

IEEE Log Number 9143002. the high attenuation that biological materials present at microwaves, which place stringent requirements on the equipment design, and the difficulty in developing imaging algorithms adapted to the high dielectric contrast of biological bodies.

The first experiments on active microwave biological imaging were carried out by Larsen and Jacobi in the late 1970's [5]. The images, which showed the internal features of an isolated canine kidney, were obtained by mechanically scanning the body with a pair of antennas and measuring the transmission coefficients between them. Since then, an important effort has been conducted to design systems to measure the scattered fields produced by biological bodies and imaging algorithms adapted to the measurement geometry to reconstruct the permittivity data [6]-[14].

Conventional microwave tomography systems are based on illuminating the body with a plane wave and measuring the scattering with a linear array of probes, in a way similar to that proposed by Mueller in acoustic diffraction tomography [15]. With plane wave illumination and the assumption of weak scattering bodies, the plane wave spectrum of the scattered field contains the information on a circular arc of the bidimensional Fourier spectral domain of the dielectric properties of the body [13]. Repeating the measurement for different directions of incidence or views, the spectral domain can be filled and inverted, obtaining an image of a cut of the body. A practical problem with this method is the need for mechanical rotation of either the body or the antenna in order to obtain measurements in different views. Although linear synthetic approaches have been proposed to avoid this problem [9], [16], they are unable to produce complete turn multiview data without rotation. An alternative proposed by Bolomey consists in using a twodimensional array to measure the scattered fields [8], with acquisition times of the order of a second. The images obtained show details of the internal dielectric structure or dielectric changes in real and phantom biological bodies [6]-[8].

Using an encircling geometry of measurement would make it possible to obtain multiview data without mechanical movement. This paper presents a first prototype 
based on a cylindrical array, whose elements can be addressed independently as emitters or receivers. This configuration allows a fast exploration of body's cut placed along the array axis, in a way similar to that of present $\mathrm{X}$-ray scanners. The electromagnetic compatibility (EMC) of this approach is critical because the strongly attenuated received fields are measured on the same array which is being used to emit a high-level illuminating signal. Therefore, carefully designed high-frequency architectures and detection techniques are necessary.

The next section of the paper shows the frequency choice as a compromise between resolution of the image and tolerable attenuation, in our prototype a working frequency of $2.45 \mathrm{GHz}$ has been adopted. Section III describes the main parts of the prototype, an efficient imaging algorithm for cylindrical geometry and the main characteristics. Section IV presents results obtained with the prototype corresponding to biological phantoms and real bodies. In the final section, the main conclusions resulting from this work are presented.

\section{Choice of Frequency}

The working frequency in imaging systems intended for biological applications is a compromise between spatial resolution and tolerable attenuation. Spatial resolution of electromagnetic images is limited by diffraction to the order of a half wavelength of the radiation in the object explored [17]; thus the resolution improves when the frequency is increased. The wavelength in biological tissues is substantially shorter than values in air because of the high water content. Water has a relative dielectric constant of the order of 70 to 80 at microwave frequencies and room temperatures. The wavelength is reduced in this medium by a factor of almost 9 in relation to air values. Therefore a theoretical resolution of several millimeters can be achieved with operating frequencies of a few gigahertz.

On the other hand, biological bodies exhibit strong attenuation at microwave frequencies because of the high content of water and conducting solutes. The attenuation increases rapidly with frequency, which limits effectively the working frequency depending on the size of the body, the illumination power, and the receiver sensitivity. For safety reasons, the illumination power density must be kept below the microwave radiation standards for continuous exposure [18]. Receiver sensitivity is linked to the integration time of each measurement and consequently is proportional to the acquisition time. Imaging usually requires the measurement of thousands of scattered field complex data, which means that in order to have acquisition times of the order of seconds the integration time must be around $1 \mathrm{~ms}$. From these considerations the useful frequency range for microwave imaging is found to be from below $1 \mathrm{GHz}$ for larger bodies such as the thorax or pelvis to several $\mathrm{GHz}$ for smaller ones such as limbs. In our case a frequency of $2.45 \mathrm{GHz}$ has been chosen, which is assigned to industrial-scientific-medical applications.
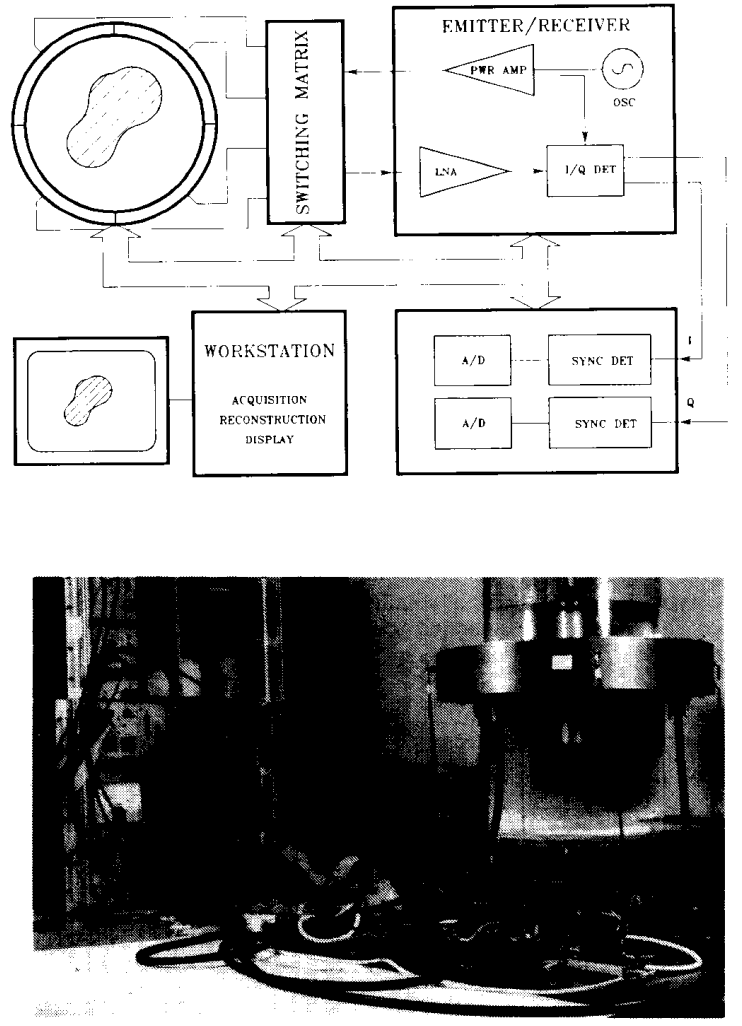

Fig. 1. Block diagram and photograph of the prototype system.

\section{A Cylindrical Prototype System}

A prototype cylindrical system has been developed to evaluate the performance and limitations of this approach and to investigate possible clinical applications. The system is based on a cylindrical array of 64 antennas equispaced on a circle $25 \mathrm{~cm}$ in diameter. Although the array is in fact circular, the name cylindrical is used because each antenna is assumed to produce an almost cylindrical wave on the illuminated region. In this way a bidimensional reconstruction algorithm has been formulated in cylindrical coordinates. The basic measurement or view consists in using an antenna of the array as emitter and receiving the scattered fields with the remaining elements. This procedure is repeated, sequentially emitting with each of the antennas to complete one turn. Thus, as many views as array elements are obtained with no movements, using a measurement method very similar to that used in the modern fan-beam X-ray scanners. However, using elements of a common array as emitters and receivers requires a complicated addressing network with severe isolation specifications.

The main parts of the prototype are the cylindrical array of 64 antennas immersed in water using a plastic cylindrical container; a microwave distribution network that directs the transmitted and received signals to a pair 


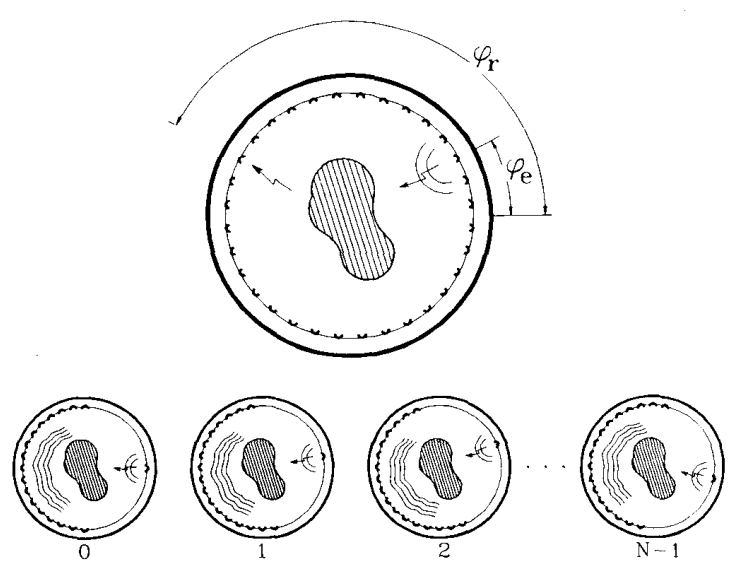

Fig. 2. Measurement procedure in a cylindrical system; $\varphi_{e}$ and $\varphi_{r}$ are the angular coordinates of the emitter and receiver antennas in operation. The object is illuminated sequentially with the elements of the array (views); for each view the scattered fields are measured.

of antennas of the array; a coherent microwave emitterreceiver; some low frequency circuitry (Fig. 1); and a 32 bit microcomputer that controls the acquisition and reconstructs and presents the image on a high-resolution color screen. These parts will now be described.

\section{A. Cylindrical Array}

The array is formed by 64 waveguides working in the usual $\mathrm{TE}_{10}$ mode opened on a cylindrical $E$-plane exponential taper. The taper shape was optimized in order to concentrate the fields through the cut of the body explored. The electric field polarization is parallel to the axis of the array. The diameter of the array is $25 \mathrm{~cm}$ but owing to the taper the useful diameter is reduced to $20 \mathrm{~cm}$. The array is fully immersed in distilled water having a complex permittivity of $\epsilon=77-j 9$ at $2.45 \mathrm{GHz}$ and room temperature; this medium is used to match the high permittivity values of biological materials. The wavelength at this frequency in water is around $14 \mathrm{~mm}$; in this way the waveguides dimensions are comparable to those used at $\mathrm{K}$ band in air.

With the $E$ plane designed, the field distribution along the axis of the array produced by an emitting element has a width of $6 \mathrm{~cm}$ measured between $-3 \mathrm{~dB}$ points, which corresponds to the thickness of the slice of the body which is actually explored.

\section{B. Distribution Network}

An antenna can be used as an emitter or a receiver, making it possible to use a unique array as emitter and receiver. A complete scattering measurement would involve addressing sequentially every antenna of the array as emitter and then measuring the scattered fields on all the remaining elements of the array, as depicted in Fig. 2. When the body to be imaged is placed in the center of the array, the field received can be considered the superposition of the incident field which would be measured without the body, plus the scattered field. The scattered field is produced by the equivalent or induced currents in the body and forms the usual input data of the reconstruction algorithms. The scattered field must be obtained by subtracting the incident field measured without the body from the total field measured once the body has been placed in the measurement region. Because of the high attenuation of microwaves when propagating through water and biological materials (attenuations in excess of 100 $\mathrm{dB}$ are usual), one soon realizes the difficulty of performing a complete measurement. First, the scattered field received by the elements close to the transmitting antenna would be so weak compared with the incident field that the total and incident fields will be almost equal, requiring an impractical dynamic range to obtain useful data. Another problem arises in considering the isolation of the array multiplexer necessary to avoid interference between the high-level transmitting and weak receiving signal paths. These problems have been solved by dividing the array into several highly isolated sections that are addressed globally as transmitter or receiver. In our case the array has been divided into four sections of 16 elements each. In order to use the same configuration of receiving elements per view, independently of which position takes the emitter element in each section, only the half array opposed to the emitter is used as the receiver. Numerical simulations have shown that usually the scattered field is mainly directed in transmission; moreover the reflected scattered fields are very difficult to measure by subtraction between the strong total and incident fields.

The 1 to 16 multiplexer of each section consists of a binary tree of SPDT diode switches. The connection between the array section ports and the transmitter and receiver is made by means of a switching matrix; however, the leakage between transmitting and receiving sections requires nonpractical isolations of the switches involved. Thus, additional ways of increasing the isolation must be adopted. In this case the problem has been solved using low-frequency modulation to distinguish the useful signals from interference. A double low-frequency amplitude modulation is used near the transmitter and receiver antennas. The detection of the received signal is synchronous with a clock running at the sum frequency of the transmitter and receiver modulators; in this way only the signals modulated in transmission and reception are correlated with the detector clock and give a nonzero output. This procedure avoids, for example, direct interference between the emitter and receiver. A low-noise amplifier has been included in the receiving path in order to obtain a noise figure at the receiver of $3 \mathrm{~dB}$.

\section{Microwave Emitter-Receiver}

The emitter consists of an oscillator phase-locked to a subharmonic stable reference, followed by a power amplifier delivering $1 \mathrm{~W}$ of $\mathrm{CW}$ signal at $2.45 \mathrm{GHz}$. The 
maximum incident power density in the measurement region of the array is less than $0.1 \mathrm{~mW} / \mathrm{cm}^{2}$, which is well below the recommended ANSI standard [18] limit for continuous exposure $\left(5 \mathrm{~mW} / \mathrm{cm}^{2}\right.$ at $\left.2.45 \mathrm{GHz}\right)$. The receiver uses a coherent homodyne $\mathrm{I} / \mathrm{Q}$ detector. A directional coupler inserted in the transmitter path provides the LO reference signal for the detector. The in-phase and quadrature outputs contain a dc component, as a consequence of detector bias and continuous wave interference, and low-frequency modulated signals. The desired signals are extracted with a pair of synchronous detectors, providing a high sensitivity to the system. The $\mathrm{dc}$ outputs of the detectors are sampled by two $13 \mathrm{bit}$ A/D converters with an integration time of approximately $1 \mathrm{~ms}$. This means that the basic dynamic range of the system is $78 \mathrm{~dB}$; however this figure can be increased by data averaging if necessary.

\section{Acquisition and Calibration}

The system is controlled by a 32 bit workstation (HP9000-320) using a parallel input/output bus and conventional logic circuitry. The acquisition consists in addressing all possible pairs of sectors, one acting as transmitter and the other as receiver. For each pair the individual elements are addressed in such a way that for each emitting element the total field is measured with the opposite half array. A field data set is a matrix of $64 \times 64$ elements where the columns are the transmitting elements and the rows the receivers; only 2112 points contain scattering data because the reflected fields are not measured. The acquisition time of the system is $3 \mathrm{~s}$; data averaging makes it possible to increase the system sensitivity at the expense of longer measurement times.

As pointed out before, the scattered fields are obtained by subtraction between total and incident fields, so stability and repeatibility are crucial in the operation of the system. Another important aspect when considering measurement systems is providing an adequate means of calibration, which has been a key factor in microwave instrumentation. To calibrate the system, an object with predictable scattering characteristics is measured and then the necessary correction coefficients for each emitterreceiver couple of elements are obtained by dividing the theoretical measurement by the actual one. A 4-cm-diameter metallic cylinder is used for this purpose; the diameter has been chosen as a compromise between scattering amplitude and relative flatness along the receiving elements.

\section{Imaging Algorithm}

Unlike $X$ rays, microwave radiation has a wavelength comparable to the size of the object; thus refraction and diffraction cannot be neglected and diffraction imaging algorithms must be used. In a cylindrical array the field produced by each element will be approximately a cylindrical wave emerging from the antenna if the field energy is assumed to be confined to the thickness of the array.
For each emitter the scattered field will be measured on other elements of the array, yielding a matrix $E_{s}\left(\varphi_{r}, \varphi_{e}\right)$, where $\varphi_{r}$ is the angular position of the receiver element and $\varphi_{e}$ the angular position of the emitter, as shown in Fig. 2 .

Using an electric field polarized axially and neglecting depolarization effects in the body, we can use an approximate scalar representation of currents and electric field [16]. Conventional algorithms are based on linear geometries of measurement and make use of first-order approximations, which means that the body is assumed to perturbate slightly the incident field. Illuminating the body with a plane wave, the plane wave angular spectrum of the scattered field contains the spectrum of the dielectric contrast of the object along circles of radius $k_{0}$ (the wavenumber of the field in the embedding reference medium) [16]. The dielectric contrast $C(\vec{r})$ is the relative difference between the complex permittivity of each point of the body $\epsilon(\vec{r})$ and the reference permittivity $\epsilon_{0}$ corresponding to the embedding medium: $C(\vec{r})=1-\epsilon(\vec{r}) / \epsilon_{0}$.

In our case the geometry of measurement is cylindrical and a similar algorithm can be obtained based on Hankel transforms; however this algorithm is difficult to implement on a digital computer and very inefficient because of the large number of Bessel functions involved. A much more efficient algorithm can be obtained by using a synthetic approach to form plane waves as a combination of cylindrical waves.

First, given the linearity of the scattering, we can synthesize a plane wave illumination in an arbitrary direction by combining the measurements of the different emitters weighted by the current distribution on the array that would produce this plane wave illumination:

$$
E_{s}\left(\varphi_{r} ; \hat{\theta}_{0}\right)=\int_{0}^{2 \pi} \tilde{E}_{s}\left(\varphi_{r}, \varphi_{e}\right) J_{a}\left(\varphi_{e} ; \hat{\theta}_{0}\right) R d \varphi_{e}
$$

where $J_{a}\left(\varphi_{e} ; \hat{\theta}_{0}\right)$ are the currents on the array producing a plane wave in the direction of the unit vector $\hat{\theta}_{0}$. On the other hand, using the reciprocity theorem, the Fourier spectrum of the induced currents $J(\vec{r})$ in the dielectric can be obtained by weighting the measurements on the array $a$ again by the current distribution that would produce a plane wave in the direction of the unit vector $-\hat{\theta}$ in the region $s$ occupied by the body [19]:

$$
\tilde{J}\left(\vec{\eta}=k_{0} \hat{\theta}\right)=\int_{S} J(\vec{r}) e^{j k_{0} \hat{\theta} \cdot \dot{r}} d \vec{r}=\int_{a} J_{a}(\varphi ;-\hat{\theta}) E_{s}(\varphi) R d \varphi
$$

where $R$ is the radius of the array. Assuming the scattering to be sufficiently low to approximate the total field within the body by the incident field (Born approximation), we can express the induced currents in the dielectric as a product of the dielectric contrast and the incident field $E_{0}$. In a lossless reference medium (real $k_{0}$ ), this product implies a shift of $k_{0} \hat{\theta}_{0}$ between the spectral 


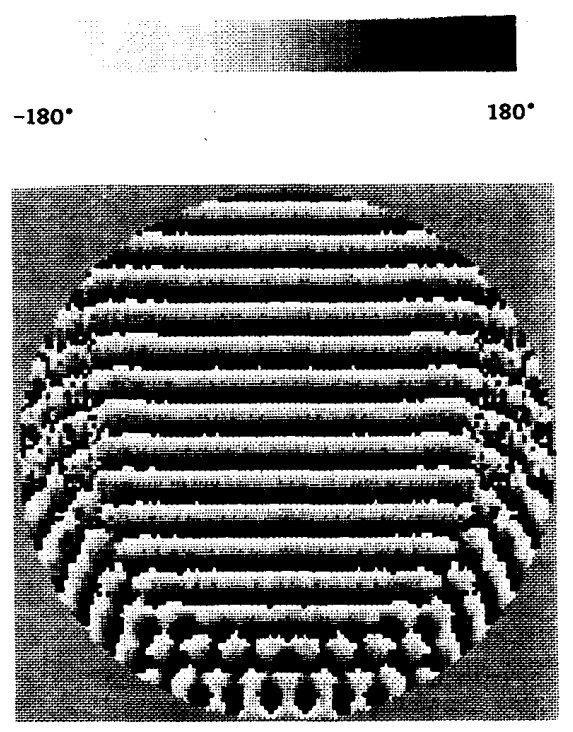

Fig. 3. Phase of a synthetically generated plane wave from cylindrical waves emerging from the elements of the array.

domains of current and contrast:

$$
\begin{aligned}
J(\vec{r}) \cong-j \omega \epsilon_{0} C(\vec{r}) E_{0}(\vec{r}) \stackrel{\mathscr{F}}{\Rightarrow} \tilde{J}(\vec{\eta}) \\
\\
\cong-j \omega \epsilon_{0} \tilde{C}\left(\vec{\eta}-k_{0} \hat{\theta}_{0}\right)
\end{aligned}
$$

where

$$
\tilde{C}(\vec{\eta})=\mathscr{F}\{C(\vec{r})\}=\int_{-\infty}^{\infty} C(\vec{r}) e^{-j \dot{\eta} \cdot \vec{r}} d \vec{r}
$$

is the bidimensional Fourier transform of the dielectric contrast $C(\vec{r})$. Combining (1), (2), and (3), we obtain finally the spectral domain of $C(\vec{r})$ on circular arcs of radius $k_{0}$ with coordinates $\vec{\eta}=k_{0}\left(\hat{\theta}-\hat{\theta}_{0}\right)$ :

$$
\begin{array}{r}
\tilde{C}\left[k_{0}\left(\hat{\theta}-\hat{\theta}_{0}\right)\right]=\frac{j}{\omega \epsilon_{0}} \int_{0}^{2 \pi} \int_{0}^{2 \pi} E_{s}\left(\varphi_{r}, \varphi_{e}\right) J_{a}\left(\varphi_{r} ;-\hat{\theta}\right) \\
\cdot J_{a}\left(\varphi_{e} ; \hat{\theta}_{0}\right) R^{2} d \varphi_{r} d \varphi_{e} .
\end{array}
$$

A similar expression was derived previously by Devaney and Beylkin for arbitrary geometries using a different approach [20]. Equation (5) is formally a double convolution of the measured scattered fields $E_{s}$ with the currents on the array $J_{a}$ that would produce a plane wave illumination. The convolution can be efficiently calculated using FFT techniques as a double product in the spectral domain between the Fourier series of $E_{s}$ and $J$ as described in [19]. Fig. 3 shows the superposition of cylindrical waves weighted by the coefficients $J_{a}(\varphi)$. Only the phase of the resulting plane wave in the measurement region is shown for simplicity. Because of water attenuation, the amplitude decays exponentially.

The image is obtained by inverse bidimensional Fourier transform once $\tilde{C}\left[k_{0}\left(\hat{\theta}-\hat{\theta}_{0}\right)\right]$ has been mapped on a rectangular grid by bilinear interpolation in a way similar
TABLE I

\begin{tabular}{lll}
\hline \multicolumn{1}{c}{ System Parameters } & \\
Frequency & $2.45 \mathrm{GHz}$ \\
Power density & $<0.1 \mathrm{~mW} / \mathrm{cm}$ \\
Useful diameter & $20 \mathrm{~cm}$ \\
Data acquisition time & $3 \mathrm{~s}$ \\
Reconstruction time & $37 \mathrm{~s}$ \\
& on HP320 \\
& \\
Spatial resolution & \multicolumn{1}{c}{ Image } & $\mathrm{mm}$ \\
Contrast resolution & $1 \%$ \\
Temperature resolution (in water) & $0.5^{\circ} \mathrm{C}$ \\
\hline
\end{tabular}

to that in linear algorithms [16]. The reconstruction time is $30 \mathrm{~s}$ on a HP9000-320 workstation.

Unfortunately the high dielectric contrast of biological bodies often causes the breakdown of the Born approximation [9], [16]. This depends on both the size and the contrast of the object. It has been found both numerically and experimentally that some biological bodies with sections of a few wavelengths can be reconstructed, revealing qualitatively their internal structure. When the size is larger, it is still possible to get useful differential reconstructions showing dielectric changes, as described in the next section. Although as shown in (2) the spectrum of the induced currents can be obtained from the scattered fields without approximations, it must be pointed out that the currents are different for each incident field and a knowledge of only an arc of a two-dimensional spectrum is not enough to produce a representative image of the currents. For this reason it is necessary to map approximately the currents into the body contrast, filling its 2-D spectrum, which is invariant with the incident field.

Another source of error arises because of the losses of the reference material (water). The algorithm described accounts for the losses except in the last step, when the image is obtained by Fourier inversion of its spectral domain using FFT under the assumption of a uniform incident field $E_{0}$. With a lossy medium, $E_{0}$ is attenuated; consequently we have access to the Laplace spectrum of the dielectric contrast [21]. It has been stated by simulations that this low-loss approximation does not cause strong errors if the losses in the body are not significantly different from those in the reference medium. Table I summarizes the main characteristics of the system developed and the imaging algorithm.

\section{Results}

One of the advantages of the calibration method presented is the possibility of obtaining an absolute calibration of the system and thus being able to reconstruct quantitatively the actual permittivity distribution of bodies. To test this possibility, a low-contrast gel cylinder was measured and reconstructed. Fig. 4 shows the image of complex permittivity in real/imaginary part in a linear gray scale. Comparing the image values with the actual gel permittivity $\epsilon_{g}=68-j 16$, the imaginary part is well reconstructed and the real part is correctly reconstructed in the external layer of the cylinder. With the present 


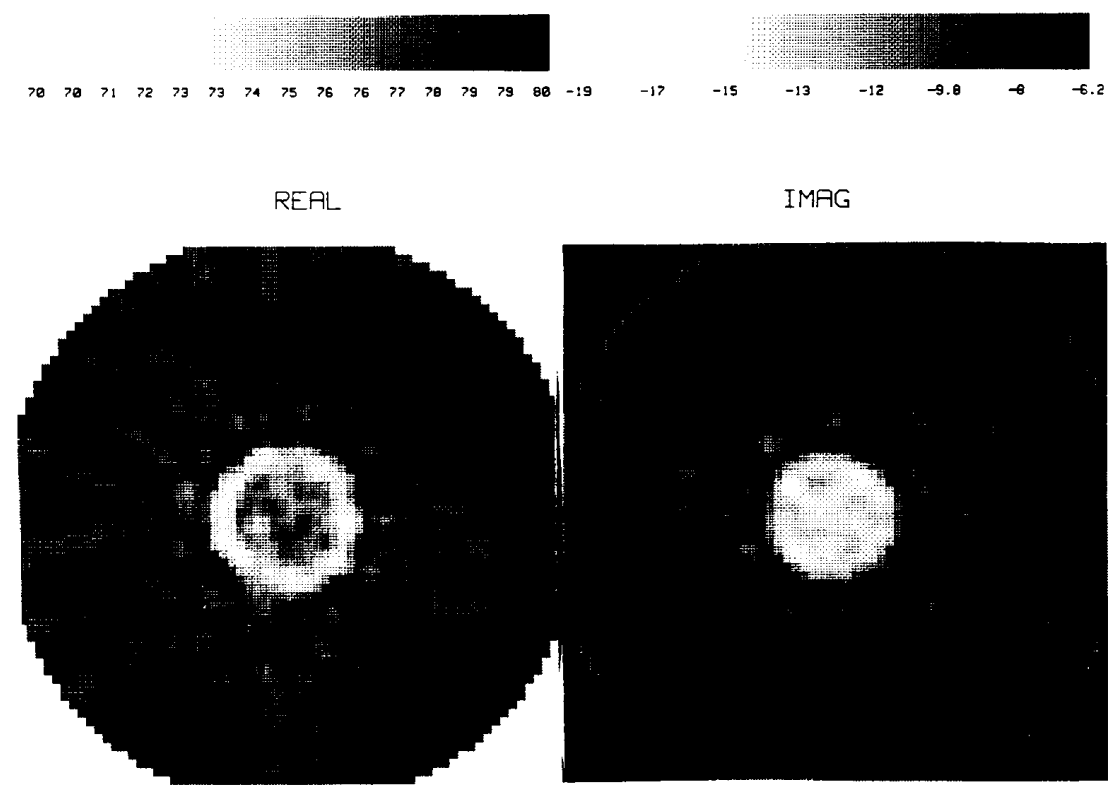

Fig. 4. Quantitative image of complex permittivity in real/imaginary part of a homogeneous gel rod with permittivity $\epsilon_{g}=68-j 16$.

algorithm, this is possible only with weak scattering objects. The experimental resolution of the system has been obtained by reconstructing two close thin metallic rods. The objects have been found separable at a minimum distance of $8 \mathrm{~mm}$. This value is close to the theoretical half-wavelength diffraction limit $(7 \mathrm{~mm})$.

With high-contrast biological bodies multiple scattering appears and the incident field is a worse approximation for the total field in the body; however the algorithm described still correctly reconstructs small section bodies. Fig. 5 shows a tomographic image of dielectric contrast amplitude corresponding to the fingers of a human hand. Fig. 6 is the diagram of a simplified arm phantom and its reconstruction (imaginary part of permittivity). Although the image is qualitative, which means that the reconstructed dielectric values are not correct, the structure of the object is successfully visualized. Fig. 7 is the reconstruction of a human forearm in vivo. The imaginary part of the permittivity shows the outer skin and fat layers, the bones radius and ulna, and some less well defined details in the muscular region with a much higher dielectric constant. This result shows that it is possible to visualize high-contrast bodies which fall outside the region of validity of the Born approximation.

The acquisition speed of the system makes it possible to monitor functional changes in a body. For this purpose differential images are formed by processing the difference in total fields produced by a reference and altered bodies. Fig. 8 shows a differential image of dielectric contrast amplitude corresponding to a blood content alteration in a human arm when a tourniquet is applied [22]. This experiment shows the potential application

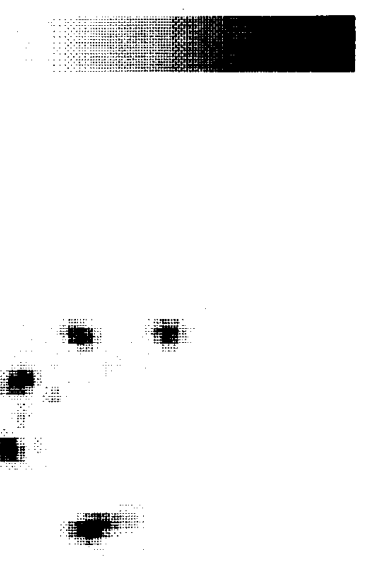

Fig. 5. Tomographic image of the fingers of a human hand.

of microwave imaging techniques to the monitoring of changes involving permittivity alterations such as irrigation. Water permittivity exhibits a high sensitivity to temperature $\left(-0.5 \% /{ }^{\circ} \mathrm{C}\right.$ in real part and $-2 \% /{ }^{\circ} \mathrm{C}$ in imaginary part), making it possible to form images of temperature changes in bodies. The temperature resolution was found experimentally forming differential images of a thin, 3-cm-diameter plastic tube filled with temperature controlled water [22]. The minimum detectable change was $0.5^{\circ} \mathrm{C}$, which corresponds to a contrast resolution of approximately $1 \%$. This sensitivity is expected to 

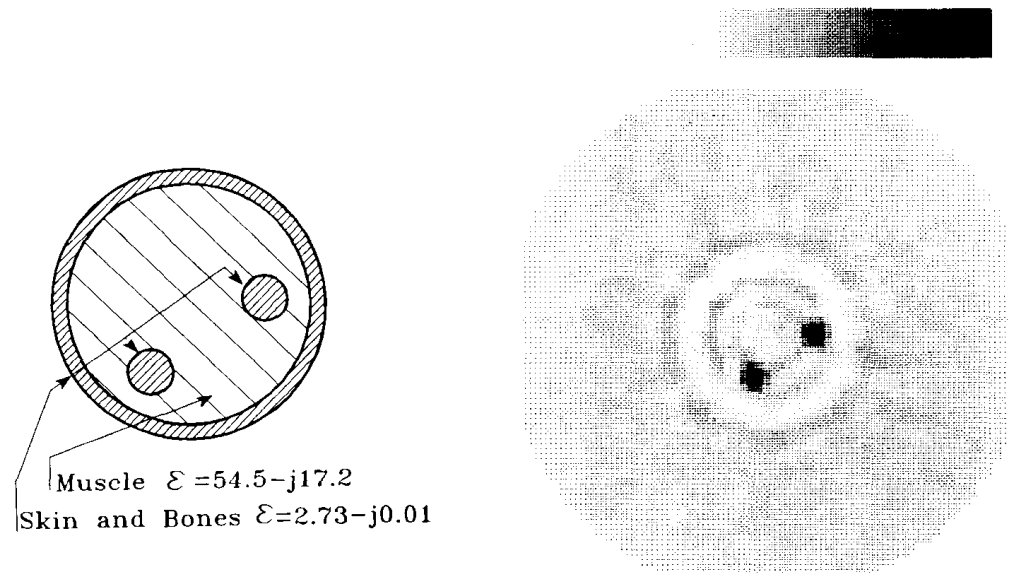

Fig. 6. Diagram of a simplified arm phantom and its reconstruction.

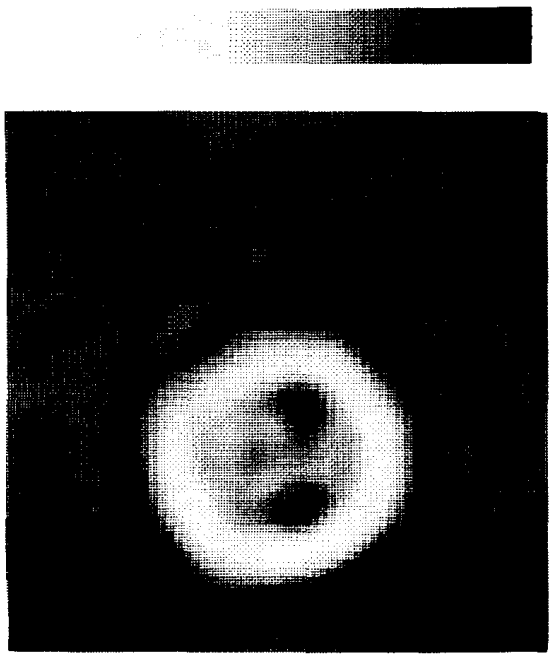

Fig. 7. Reconstruction of a human forearm in civo.

deteriorate in biological media because of their higher contrast and losses. Moreover the actual thermal sensitivity of permittivity is expected to depend on the constitutive materials of the tissue and possible thermoregulatory mechanisms with impact on permittivity, such as blood flow variations. Further research is needed to evaluate the actual thermal sensitivities in this application.

Differential techniques can be applied to large bodies when absolute images do not show their internal features owing to the breakdown of first-order approximations [23]. Fig. 9 shows the differential reconstruction of a human head phantom when a white matter equivalent medium with permittivity $\epsilon=49-j 18.5$ (close to data reported in [3]) is replaced with water with a higher permittivity. The image of the real part of the dielectric contrast shows well the change, although the absolute image shows only the contour of the phantom because of
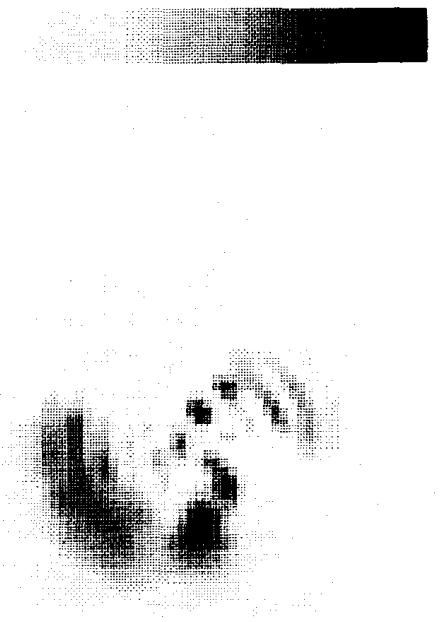

Fig. 8. Differential image corresponding to a blood content alteration in the arm shown in Fig. 7 when a tourniquet is applied.

its size and high contrast. A higher sensitivity would be necessary to visualize small permittivity changes that could be related to cerebral activity.

\section{Conclusions}

A cylindrical system for active microwave tomography has been constructed and evaluated. The system allows a 64 view scan in $3 \mathrm{~s}$ using microwave multiplexers. The thickness of the explored cut of the object is $6 \mathrm{~cm}$ wide; this value could be possibly reduced using a lens to focus the energy in the array plane. The electromagnetic compatibility of the system is critical, and the desired signal has been isolated from the interference by subdividing the array and using a double low-frequency synchronous detection. 

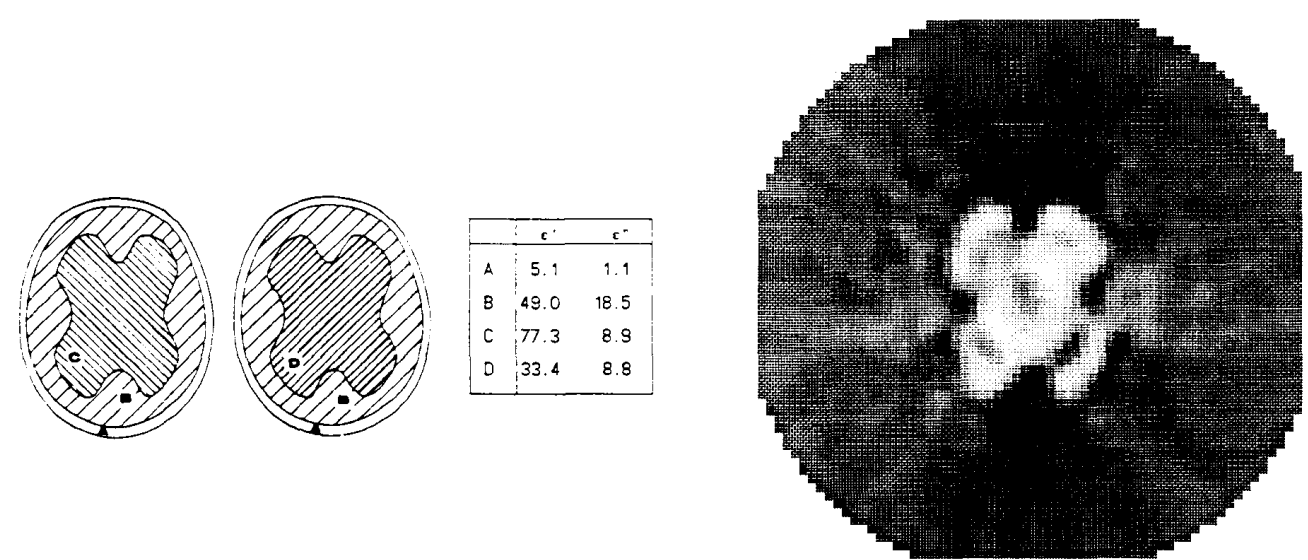

Fig. 9. Differential reconstruction of a human head phantom when a white matter equivalent medium is substituted by water with higher permittivity.

The imaging algorithm is based on the synthesis of plane waves and can be efficiently implemented on a computer. The algorithm is based on a bidimensional scalar formulation of the fields, weak scattering, and low-loss approximations. An absolute calibration procedure has been developed which allows quantitative imaging of weak scattering bodies. High contrast bodies can be qualitatively imaged providing their size is below five or six wavelengths. New algorithms more adapted to the high dielectric contrast of biological bodies would make it possible to overcome this limitation. Differential imaging is able to reconstruct dielectric changes in the body, even if electrically large, by processing the variations measured in the total or scattered fields. Some experimental results with phantoms and bodies in vico have shown the capability of microwave tomography to image the dielectric structure or functional changes of biological bodies.

Compared with other conventional imaging methods, microwave tomography has several advantages. First, the radiation is nonionizing and can be considered virtually safe using low-power illumination. Present acquisition times of the order of a few seconds could be reduced in the future, making it possible to perform dynamic studies of tissue movements as in the heart, joints, blood vessels, etc., or to visualize dielectric changes caused by physiological and biochemical alterations. Finally, the high cost and environmental constraints imposed by magnetic resonance imaging, X-ray CT, and positron emission tomography systems limit their clinical availability. Microwave systems have a lower cost and can be compatible with other clinical equipment. These advantages are expected to play an important role in the future development and clinical acceptance of this technique.

Although the system has been conceived for biomedical applications at $2.45 \mathrm{GHz}$, it could be easily adapted for industrial or nondestructive-testing (NDT) purposes scal- ing the dimensions and frequency, depending on the sizes and dielectric characteristics of the objects.

\section{ACKNOWLEDGMENT}

The authors are greatly indebted to E. de los Reyes, M. Ferrando, M. Baquero, M. Hawley, and H. Almirall for their contributions and helpful discussions. Special thanks go to A. Cano, J. Giner, and J. M. Haro for their support in the constructive aspects of the prototype.

\section{REFERENCES}

[1] D. C. Barber, B. H. Brown, and I. L. Freeston, "Imaging spatia distributions of resistivity using applied potential tomography," Electron. Lett., vol. 19. no. 22, pp. 933-934, 1983

[2] L. E. Larsen et al., Eds., Medical Applications of Microwave Imag. ing. New York: IEEE Press, 1986, pp. 148-166.

[3] M. A. Stuchly and S. S. Stuchly, "Dielectric properties of biologica substances-Tabulated," J. Microwate Power, vol. 15, no. 1, pp. $19-25,1980$.

[4] H. P. Schwan, "Electrical properties of tissue and cell suspensions," Adr. Biol. and Med. Phys., vol. 5, pp. 147-209, 1957.

[5] L. E. Larsen and J. H. Jacobi, "Microwave scattering parameter imagery of an isolated canine kidney," Med. Phys., vol. 6, no. 5, pp. 394-403, Sept. /Oct. 1979.

[6] G. Peronnet, Ch. Pichot, J. Ch. Bolomey, and L. Jofre, "A microwave diffraction tomography system for biomedical applications," in Proc. 13th European Microwace Conf. (Nurnberg), Sept. 1983 pp. 529-533.

[7] J Ch. Bolomey, L Jofre Ch. Pichot, and G. Peronnet "Microwave diffraction tomography for biomedical applications," IEEE Trans. Microwale Theory Tech., vol. MTT-30, pp. 1988-2000, Nov. 1982.

[8] Ch. Pichot, L. Jofre, G. Peronnet, and J. Ch. Bolomey, "Active microwave imaging of inhomogeneous bodies," IEEE Trans. Antennas Propagat., vol. AP-33, pp. 416-425, Apr. 1985.

[9] H. Ermert and M. Dohlus, "Microwave-diffraction-tomography of cylindrical objects using 3-dimensional wave fields," ntzArchiv, Bd 8 , H. 5, pp. $111-117,1986$

[10] A. P. Anderson and M. F. Adams, "Holographic and tomographic imaging with microwaves and ultrasound," in Inverse Methods in Electromagnetic Imaging, D. Reidel, pp. 1077-1105, 1985.

[11] S. J. Foti, R. P. Flam, J. F. Aubin, L. E. Larsen, and J. H. Jacobi, "A water-immersed microwave phased array system for interroga- 
tion of biological targets," in Medical Applications of Microwate Imaging, L. E. Larsen and J. H. Jacobi, Eds. New York: IEEE Press, 1986 , pp. 148-166.

[12] L. Jofre et al. "A cylindrical system for quasi-real-time microwave tomography," in Proc. 16th European Microwale Conf. (Dublin), Sept. 1986, pp. 599-604.

[13] S. X. Pan and A. C. Kak, "A computational study of reconstruction algorithms for diffraction tomography: Interpolation versus filtered backpropagation," IEEE Trans. Acoustics, Speech. Signal Process. vol. ASSP-31, pp. 1962-1275, Oct. 1983.

[14] D. K. Ghodgaonkar, O. P. Ghandhi, and M. J. Hagmann, “Estimations of complex permittivities of three-dimensional inhomogeneous bodies," IEEE Trans. Microwace Theory Tech., vol. MTT-31, pp. 442-446, June 1983.

[15] R. K. Mueller, M. Kaveh, and G. Wade, "Reconstructive tomography and application to ultrasonics," Proc. IEEE, vol. 67. pp. 567-587. Apr. 1979

[16] M. Slaney and A. C. Kak, "Imaging with diffraction tomography," Purdue University, School of Elect. Eng., Tech. Rep. TR-EE 85-5, 1985

[17] J. W. Goodman, Introduction to Fourier Optics. New York: McGraw-Hill, 1968

[18] American National Standards Institute ANSI C95.1-1982 "Safety levels with respect to human exposure to radiofrequency electromagnetic fields, $300 \mathrm{kHz}$ to $100 \mathrm{GHz}$," IEEE. New York, NY, 10017 .

[19] J. M. Rius, M. Ferrando, L. Jofre, and A. Broquetas, "Microwave tomography: An algorithm for cylindrical geometries." Electron. Lett.. vol. 23, no. 11 . pp. 564-565, 1987.

[20] A. J. Devaney and G. Beylkin. "Diffraction tomography using arbitrary emitter and receiver surfaces." Ultrason. Imaging. no. 6. pp. $181-193.1984$

[21] M. Azimi and A. C. Kak, "Multiple scattering and attenuation phenomena in diffraction imaging." Purdue Univ., School of Elect. Eng., Tech. Rep. TR-EE 85-4, 1985.

[22] L. Jofre et al., "Medical imaging with a microwave tomographic scanner," IEEE Trans. Biomed. Eng., vol. BME-37. pp. 303-312. Mar. 1990.

[23] A. Broquetas et al., "Temperature and permittivity measurements using a cylindrical microwave imaging system," in Proc. 17th European Microwate Conf., (Rome), 1987. pp. 892-895.

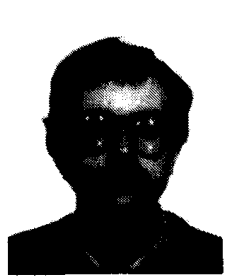

Antoni Broquetas ( $\mathrm{S}^{\prime} 84-\mathrm{M}^{\prime} 90$ ) was born in Barcelona, Spain, in 1959. He received the Ingeniero degree in telecommunication engineering from the Universitat Politècnica de Catalunya (U.P.C.) in 1985, and the Doctor Ingeniero degree in 1989 in telecommunication engineering for his work in microwave tomography.

In 1984 he joined the Electrophysics group of the U.P.C. working in microwave systems and digital radio links. In 1986 he was a research assistant at Portsmouth Polytechnic (U.K.) in volved in propagation studies. Currently he is Associate Professor a U.P.C., where he is engaged in research on microwave imaging and radar

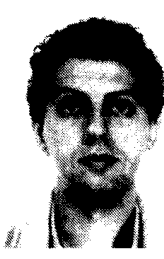

Jordi Romeu ( $\mathrm{S} 88$ ) was born in Barcelona, Spain, in 1962 . He received the Ingeniero degree in telecommunication engineering in 1986 from the Universitat Politècnica de Catalunya.

In 1985 he joined the Antenna-MicrowaveRadar group of the Signal Theory and Communications Department there. His research deals with microwave imaging, antenna near field measurements, and antenna diagnostic areas.

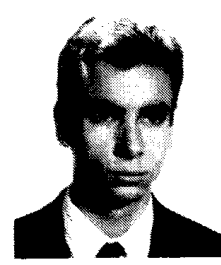

Juan M. Rius ( $\mathrm{S} 89$ ) was born in Barcelona. Spain, in 1963. He received the Ingeniero degree in telecommunication engineering from the Universidad Politècnica de Catalunya (U.P.C.) in 1987.

In 1985 he joined the Electrophysics group at U.P.C. working on cylindrical geometry algorithms for microwave tomography. Currently he is Associate Professor at the Telecommunication Engineering School of U.P.C. and is involved in electromagnetic scattering techniques for radar cross. section numerical prediction.

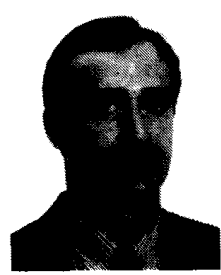

Antonio R. Elias-Fuste ( $\mathrm{S}^{\prime} 81-\mathrm{M}^{\prime} 82$ ) was born in Cervera, Spain, in 1954 . He received the Ingeniero and Doctor Ingeniero degrees in telecommunication engineering, both from the Universitat Politècnica de Catalunya (U.P.C.), in 1978 and 1982 . respectively.

In 1976 he joined the Antenas-MicroondasRadar Group of the Department of Signal Theory and Communications of the Escuela Técnica Superior de Ingenieros de Telecomunicación de Barcelona, where his work dealt with microwave characterization and RF systems design. He became Associate Professo in 1985 and Professor in 1990 at U.P.C., engaged in research in the field of radar. He is author and coauthor of several reports for industry and of papers published in technical journals and conference proceedings.

Dr. Elias-Fusté is an AOC member. Currently, he is Chairman of the AES Spanish Chapter.

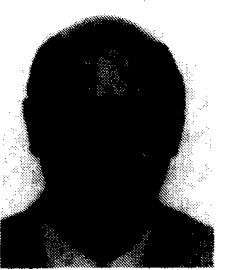

Angel Cardama (S'67-M'73) was born in Santiago. Spain, on May 13, 1944. He received the Ingeniero de Telecomunicación degree from the Universidad Politécnica de Madrid, Madrid, Spain, in 1968, and the Sc.M. and Ph.D. degrees in electrical engineering from Brown University, Providence, RI in 1970 and 1973 , respectively.

In 1972 he joined the faculty of the E.T.S.I. de Telecomunicación at the Polytechnic University of Catalonia. Barcelona, Spain, where he holds the position of Professor. His research interests range from propagation in optical fibers, high frequency aperture and array antennas, and near-field antenna scanning systems to the design of microwave imaging systems and radar antennas.

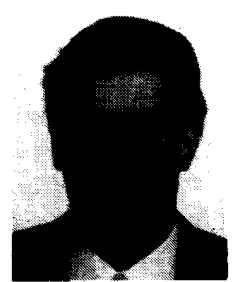

Luis Jofre (S`79-M'83) was born in Mataró Spain. in 1956. He received the Ingeniero and Doctor Ingeniero degrees in telecommunication engineering, both from the Universitat Politècnica de Catalunya (U.P.C.) in 1978 and 1982, respectively.

In 1978 he was a Research Assistant in the Electrophysics group at U.P.C., where he worked on the analysis and near-field measurement of antennas. In 1981 he joined the Ecole Supérieure d'Electricité in Paris, where he was involved in microwave imaging techniques for biomedical applications. During the period 1986-1987 he was a Visiting Fulbright Scholar at the Georgia Institute of Technology, Atlanta, working on antenna measurement and electromagnetic imaging. He is currently Professor and Director of the Telecommunication Engineering School at U.P.C., where he is engaged in research on antennas and electromagnetic scattering and imaging, both numerical and experimental aspects. 